\title{
Crosstalk Noise Model for Shielded Interconnects in VLSI-based Circuits
}

\author{
Junmou Zhang and Eby G. Friedman \\ Department of Electrical and Computer Engineering \\ University of Rochester \\ Rochester, New York 14627-0231
}

\begin{abstract}
Placing shields around a victim signal line is a common way to enhance signal integrity while minimizing delay uncertainty. An analytic model of the peak noise is developed for shielded interconnects based on a pseudo- $2 \pi R C$ model. A design methodology for inserting shields between coupled interconnects to reduce crosstalk noise is presented.
\end{abstract}

\section{INTRODUCTION}

As feature sizes are decreased to deep submicrometer dimensions, coupling capacitances can significantly affect circuit performance due to decreased interconnect spacing and increased interconnect thickness. Shielding in high speed digital circuits is an effective and common way to reduce crosstalk noise and signal delay uncertainty. A common method of shielding is placing ground or power lines at the sides of a victim signal line to reduce noise and delay uncertainty. The crosstalk between two coupled interconnects is often neglected when a shield is inserted, significantly underestimating the coupling noise. The crosstalk noise between two shielded interconnects can produce a peak noise of $15 \%$ of $V_{d d}$ in a $0.18 \mu \mathrm{m}$ CMOS technology. An accurate estimate of the peak noise for shielded interconnects is therefore necessary.

In Section II, an analytic model of the peak noise is developed for shielded interconnects based on a proposed pseudo$2 \pi R C$ model with an average error of $4.4 \%$ as compared to SPICE. In Section III, a design methodology for inserting a shield between coupled interconnects to reduce crosstalk noise is presented. Some conclusions are offered in Section IV.

\section{Analytic Model of Crosstalk}

An interconnect structure composed of two shielded signal lines is shown in Fig. 1. The victim signal line is shielded by a ground or power line from the aggressor signal line.

A proposed pseudo- $2 \pi R C$ model is used to model this interconnect structure, which is different from a standard $2 \pi R C$ model by shifting the coupling capacitances at the receiver ends to the middle nodes, as shown in Fig. 2. In a standard $2 \pi R C$ model, the coupling capacitance at the receiver ends are shorted to ground, significantly underestimating the coupling noise.

This research is supported in part by the Semiconductor Research Corporation under Contract No. 99-TJ-687 and No. 2003-TJ-1068, the DARPA/ITO under AFRL Contract F29601-00-K-0182, grants from the New York State Office of Science, Technology \& Academic Research to the Center for Advanced Technology - Electronic Imaging Systems and to the Microelectronics Design Center, and by grants from Xerox Corporation, IBM Corporation, Intel Corporation, Lucent Technologies Corporation, and Eastman Kodak Company.

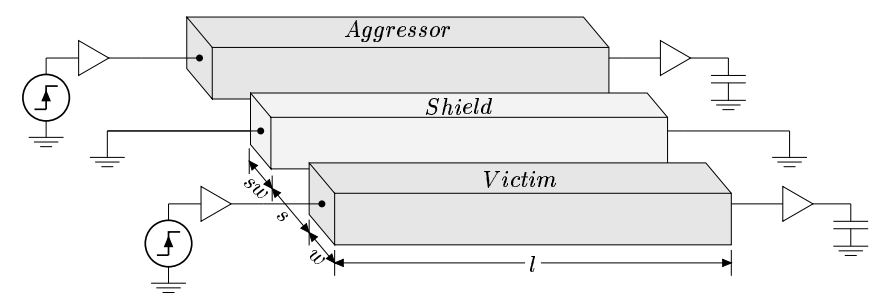

Fig. 1. Interconnect structure composed of two shielded signal lines

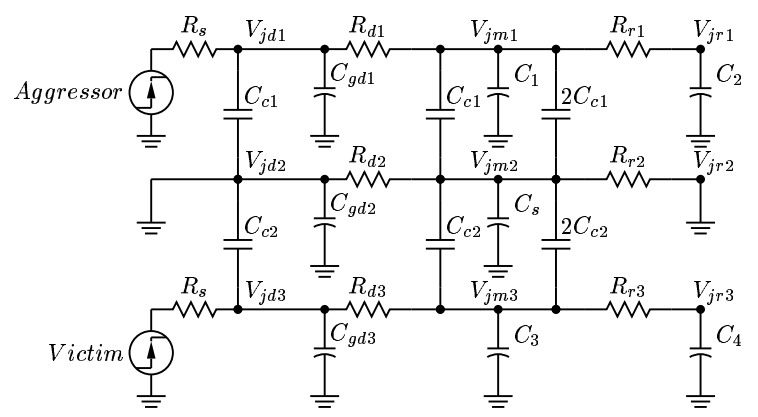

Fig. 2. Equivalent circuit model of the pseudo- $2 \pi R C$ model

Using a dominant pole truncation approximation [1], the transfer functions can be modeled as

$$
H(s) \equiv \frac{V_{j m 2}(s)}{V_{a g g}(s)} \frac{V_{j r 3}(s)}{V_{j m 2}(s)}=\frac{s^{2} t_{x 1} t_{x 2}}{\left(1+s t_{y 1}\right)\left(1+s t_{y 2}\right)} .
$$

$t_{x 1}, t_{y 1}, t_{x 2}$, and $t_{y 2}$ are

$$
\begin{aligned}
t_{x 1}= & \frac{3}{2} C_{c 1} R_{d s} \\
t_{y 1}= & R_{s 1}\left(C_{1}+C_{2}+C_{5}+3 C_{c 1}\right)+R_{d 1}\left(C_{1}+C_{2}+3 C_{c 1}\right) \\
& +R_{r 1} C_{2}+R_{s 3}\left(C_{3}+C_{4}+C_{6}+3 C_{c 2}\right)+R_{r 3} C_{4} \\
& +R_{d 3}\left(C_{3}+C_{4}+3 C_{c 2}\right)+\frac{1}{2} R_{d s}\left(C_{s}+3 C_{c 1}+3 C_{c 2}\right),(3) \\
t_{x 2}= & 3\left(R_{s 3}+R_{d 3}\right) C_{c 2} \\
t_{y 2}= & R_{s 3}\left(C_{3}+C_{4}+C_{6}+3 C_{c 2}\right)+R_{r 3} C_{4} \\
& +R_{d 3}\left(C_{3}+C_{4}+3 C_{c 2}\right)
\end{aligned}
$$

where $C_{5}=C_{g d 1}+C_{c 1}$ and $C_{6}=C_{g d 3}+C_{c 2}$. The physical meaning of $t_{x 1}, t_{y 1}, t_{x 2}$, and $t_{y 2}$ is

$t_{x 1} \quad R C$ delay of the shield line, the coupling capacitance $C_{x 1}$ times the effective resistance from node $V_{j m 2}$ to ground.

$t_{y 1} \quad$ the sum of the Elmore delays of all three nets.

$t_{x 2} \quad R C$ delay of the victim line, the coupling capacitance $C_{x 2}$ times the effective resistance from node $V_{j m 3}$ to ground.

$t_{y 2} \quad$ Elmore delay of the victim line. 
For an aggressor with a ramp input signal with a normalized power supply $V_{d d}$ and a transition time $t_{r}$, the coupling noise in the victim line is

$$
V_{j r 3}(t)= \begin{cases}\frac{t_{x 1} t_{x 2}}{t_{r}\left(t_{y 1}-t_{y 2}\right)}\left(e^{-t / t_{y 1}}-e^{-t / t_{y 1}}\right), & t \leq t_{r} \\ \frac{t_{x 1} t_{x 2}}{t_{r}\left(t_{y 1}-t_{y 2}\right)}\left(a e^{-t / t_{y 1}}-b e^{-t / t_{y 2}}\right), & t>t_{r}\end{cases}
$$

where $a=\left(1-e^{t_{r} / t_{y 1}}\right)$ and $b=\left(1-e^{t_{r} / t_{y 2}}\right)$.

To determine the peak noise, (6) and (7) are differentiated with respect to $t$ and set equal to zero. The times at which the peak noise occur are

$$
\begin{aligned}
& t_{p e a k 1}=\frac{t_{x 1} t_{x 2}}{\left(t_{y 1}-t_{y 2}\right)} \ln \left(t_{y 1} / t_{y 2}\right), \quad 0<t_{p e a k 1}<t_{r} \\
& t_{\text {peak } 2}=\frac{t_{x 1} t_{x 2}}{\left(t_{y 1}-t_{y 2}\right)} \ln \left(b t_{y 1} / a t_{y 2}\right), \quad t_{\text {peak } 2}>t_{r} \\
& t_{p e a k 3}=t_{r} .
\end{aligned}
$$

The peak noise for a shielded interconnect, therefore, is

$$
V_{j r 3}\left(t_{\text {peak }}\right)=\max \left\{V_{j r 3}\left(t_{\text {peak } 1}\right), V_{j r 3}\left(t_{\text {peak } 2}\right), V_{j r 3}\left(t_{r}\right)\right\} \text {. }
$$

The peak noise obtained from (11) is compared to SPICE, and exhibits an average error of $4.4 \%$. The coupling noise for shielded interconnect increases with longer lines (see Fig. 3), and decreases with increasing shield width (see Fig. 4) and the physical separation between the signal line and the shield line. The signal line width, however, has little effect on the coupling noise.

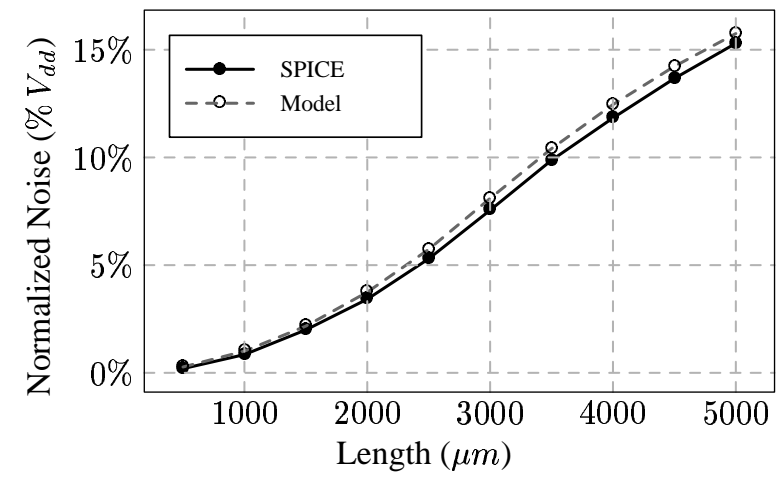

Fig. 3. Comparison of coupling noise using SPICE with the analytic model for varying lengths (the maximum error is $9.5 \%$ )

\section{Shield Insertion Methodology}

The technique of increasing the physical separation as compared to inserting a shield to reduce crosstalk noise is shown in Fig. 4. The reduction in noise achieved by inserting a shield is much greater than by increasing the physical separation. If the space between the signal lines is sufficient for a minimum width shield line, inserting a shield is more efficient in reducing noise than increasing the physical separation.

A shield line is not an ideal ground because of the parasitic resistance of the line which causes noise to couple to the victim signal line. As shown in Fig. 5, the greater the number of connections tieing the shield line to the power/ground grid, the smaller the coupling noise on the victim signal. In order

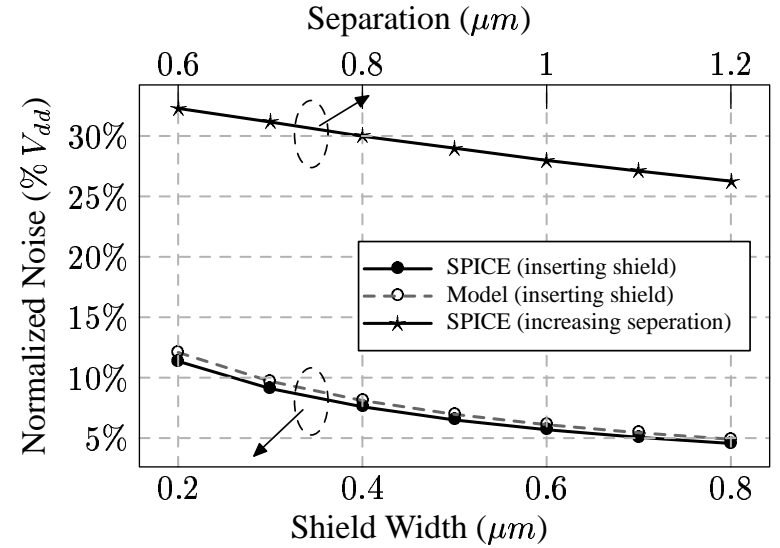

Fig. 4. Comparison of noise reduction techniques by either inserting a shield (bottom axis in the graph) or increasing the physical separation (top axis in the graph).

to satisfy the coupling noise constraints, a minimum or greater number of ground connections is required.

For a target noise constraint $V_{\max }$, the minimum number of ground connections for a shielded interconnect structure with length $l$ is

$$
N_{\text {connections }}=\frac{l}{l_{e q}},
$$

where $l_{e q}$ is the maximum length which satisfies

$$
V_{\text {noise }}\left(l_{e q}\right)<V_{\max } \text {. }
$$

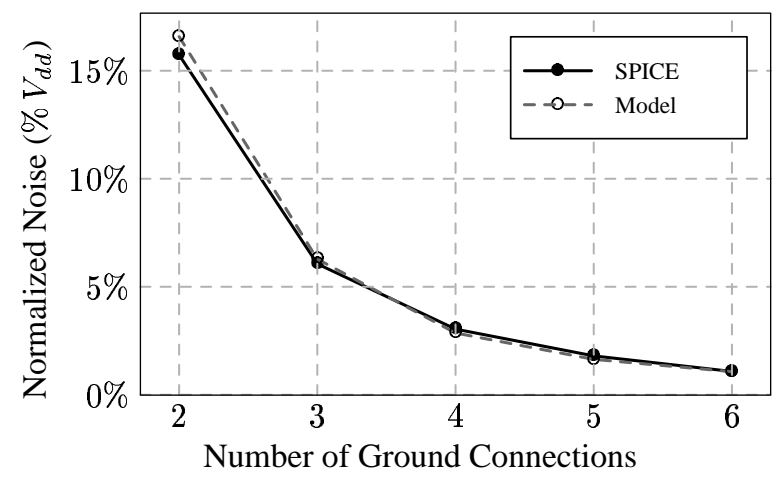

Fig. 5. The peak noise decreases with increasing number of ground connections.

\section{CONCLUSIONS}

An analytic model of the peak noise for coupled $R C$ interconnects with a shield between the lines is proposed in this paper. The peak noise model exhibits an average error of $4.4 \%$ as compared to SPICE. A methodology to insert shields between coupled interconnects is presented.

\section{REFERENCES}

[1] A. Vittal, L. H. Chen, S. M. Marek, K.-P. Wang, and X. Yang, "Crosstalk in VLSI Interconnections," IEEE Transactions on Computer-Aided Desgin of Integrated Circuits and Systems, Vol. 18, No. 12, pp. 18171824, December 1999. 